Copyright 2013 Society of Photo-Optical Instrumentation Engineers. One print or electronic copy may be made for personal use only. Systematic reproduction and distribution, duplication of any material in this paper for a fee or for commercial purposes, or modification of the content of the paper are prohibited.

https://doi.org/10.1117/12.2033945

\title{
Tailored modal gain in a multi-mode erbium-doped fiber amplifier based on engineered ring doping profiles
}

\author{
Cang Jin*, Bora Ung, Younès Messaddeq, and Sophie LaRochelle \\ Centre d'Optique, Photonique et Laser (COPL), Université Laval, Québec, QC, Canada, G1V 0A6
}

\begin{abstract}
Various space division multiplexing (SDM) schemes are currently investigated as a way to overcome the capacity limit of data links. We here focus on mode division multiplexing (MDM) in a multi-mode fiber (MMF). Towards long-haul data transmission, for which signal amplification is a key enabling component, we investigate a simple approach to precisely control the mode-dependent gain (MDG) between the co-propagating $\mathrm{LP}_{01}$ and $\mathrm{LP}_{11}$ modes of an erbiumdoped few-mode fiber amplifier (MM-EDFA) by engineering a multi-ring doping profile. In practice, the mode dependent loss of the few-mode transmission fiber must be taken into account in order to equalize the gain for all modal channels. In a step towards practical implementation of MM-EDFA for long-haul SDM, we extend the single ring doping approach to incorporate multi-ring and multi-level doping. Through numerical simulations we study the optimization of the width and doping level of each ring so as to control the MDG. We further discuss the possibility of modal gain equalization through zero-differential modal gain (ZDMG) points in a single stage MM-EDFA, or via tuning of pump powers in a dual-stage MM-EDFA configuration.
\end{abstract}

Keywords: Mode-multiplexing, multi-mode fiber amplifier, ring doping, mode-dependent gain, mode-dependent losses, spatial multiplexing.

\section{INTRODUCTION}

The exponential growth of data traffic in recent years has by now practically exhausted all known means (e.g. wavelength and polarization multiplexing, and higher-order modulation formats) of increasing the capacity limit in single-mode fibers (SMFs) [1]. Spatial multiplexing is seen as a promising route to overcome the expected capacity crunch while minimizing the energy cost per bit sent $[2,3]$. This is made possible by introducing a new degree of freedom through which one can multiplex several data links within a single fiber strand by either one of two general schemes: a multimode fiber - where each eigenmode corresponds to an independent channel [4-7] - or in a multi-core fiber where each core represents a link $[8,9]$. The present work relates to the former embodiment known as modedivision multiplexing (MDM).

Also at the heart of any practical implementation of spatial multiplexing schemes for long-haul transmission, lies the development of high-performance and cost-effective inline optical amplifiers [2]. The difficulty of achieving equal gain across all channels in a multimode erbium-doped fiber amplifier (MM-EDFA) has been discussed in [10, 11]. To circumvent this problem, active methods based on controlling the input pump conditions have been proposed [12, 13]. However, active methods relying on the injection of multiple pumps in different modes and a delicate control of their relative strengths can potentially increase the system complexity, hence the cost-per-bit.

A passive method for gain equalization based on a ring-doping approach [e.g. Figure 1(a)] was met with promising early results: $20 \mathrm{~dB}$ gain with a differential modal gain (DMG) of less than $3 \mathrm{~dB}$ has been demonstrated for the $\mathrm{LP}_{11}$ and $\mathrm{LP}_{21}$ mode groups in [14] and for the $\mathrm{LP}_{01}$ and $\mathrm{LP}_{11}$ mode groups in [15], while $12.5 \mathrm{~dB}$ gain with $1 \mathrm{~dB}$ gain excursion between the $\mathrm{LP}_{01}$ and $\mathrm{LP}_{11}$ modes was demonstrated in [16]. The same method was recently extended to include a small doped core in the center of a doped ring MM-EDFA in which four signal modes $\left(\mathrm{LP}_{01}, \mathrm{LP}_{11}, \mathrm{LP}_{21}, \mathrm{LP}_{02}\right)$ were predicted to achieve gains over $20 \mathrm{~dB}$ using two pump modes $\left(\mathrm{LP}_{01}\right.$ and $\left.\mathrm{LP}_{41}\right)$, although noise and modal coupling were neglected in the analysis [17].

In this paper we further extend this passive approach towards multiple doped rings [see Figure 1(b-c)] and demonstrate superior control over the mode-dependent gain (MDG) of a MM-EDFA supporting two signal mode groups ( $\mathrm{LP}_{01}$ and $\mathrm{LP}_{11}$ ) at $1550 \mathrm{~nm}$ and with $-13 \mathrm{dBm}$ input power each, while using a single $\mathrm{LP}_{01} 980 \mathrm{~nm}$ pump. Our numerical analysis

*cang.jin.1@ulaval.ca; http://www.copl.ulaval.ca/

Photonics North 2013, Pavel Cheben, Jens Schmid, Caroline Boudoux, Lawrence R. Chen, André Delâge, Siegfried Janz, Raman Kashyap, David J. Lockwood, Hans-Peter Loock, Zetian Mi, Eds., Proc. of SPIE Vol. 8915 89150A · @ 2013 SPIE · CCC code: 0277-786X/13/\$18 · doi: 10.1117/12.2033945 
fully takes into account the effect of amplified spontaneous emission (ASE) noise and predicts over $20 \mathrm{~dB}$ average gain for both mode groups with a DMG less than $1 \mathrm{~dB}$ and average noise figures (NF) below $5 \mathrm{~dB}$. Our analysis suggests that the proposed method should allow precise control over the gain equalization, thus opening a variety of practical working points for MM-EDFAs. We also discuss how such multi-ring multi-level doped fibers can be used in a two-stage EDFA configuration so as to enable dynamic control of the MDG via tuning of the input pump power in each stage. Finally, an argument is made towards multi-ring doped profiles for managing MDG in MM-EDFAs supporting more than two mode groups, and orbital angular momentum (OAM) modes.

\section{THEORY AND MULTI-RING DOPING PROFILES}

The operation of an erbium doped fiber amplifier can be described, as is well known, by a set of power propagation equation and rate equation [18]. The former describes the power intensity, either pump or signal, evolution through the doped fiber; while the latter updates the population densities of upper and lower level along the doped fiber, as follows:

$$
\begin{gathered}
\frac{d P_{m}(z)}{d z}=u_{m} \sigma_{e m} \int_{0}^{2 \pi} \int_{0}^{\infty} i_{m}(r, \varphi) n_{2}(r, \varphi, z) \cdot r d r d \varphi \cdot\left[P_{m}(z)+l \cdot h v_{m} \Delta v_{m}\right] \\
-u_{m} \sigma_{a m} \int_{0}^{2 \pi} \int_{0}^{\infty} i_{m}(r, \varphi) n_{1}(r, \varphi, z) \cdot r d r d \varphi \cdot P_{m}(z)-\alpha_{m} P_{m}(z) \\
n_{2}(r, \varphi, z)=\frac{\sum_{m} \frac{\sigma_{a m} i_{m}(r, \varphi) P_{m}(z)}{h v_{m}}}{\sum_{m} \frac{\sigma_{a m} i_{m}(r, \varphi) P_{m}(z)}{h v_{m}}+\sum_{m} \frac{\sigma_{e m} i_{m}(r, \varphi) P_{m}(z)}{h v_{m}}+\frac{1}{\tau}} \cdot \rho(r)
\end{gathered}
$$

where $m$ indicates the pump, signal or ASE channel, $u$ denotes the propagation direction ( 1 or -1$), \sigma_{e}$ and $\sigma_{a}$ are respectively the emission and absorption cross sections, $i_{m}(r, \varphi)$ is the normalized beam intensity profile, $n_{2}$ and $n_{1}$ are the upper and lower population level densities, $l$ indicates the number of polarization modes, variables $v$ and $\Delta v$ designate respectively the optical frequency and the ASE channel bandwidth centered at $v, h$ is the Planck constant, $\alpha$ stands for the background loss and $\tau$ is the lifetime of the metastable level of the erbium ions. Conservation is locally enforced on the population densities, $\rho=n_{1}+n_{2}$, where $\rho$ is total erbium ion population density.

In our analysis, we considered a few-mode step-index fiber that supports two modes group: $\mathrm{LP}_{01}$ and $\mathrm{LP}_{11}$. To account for the large dissimilarity in the field distributions of the modes and the ensuing spatial mode competition, the transverse cross section in our numerical model was discretized using the multi-layer approach described in [19]. Based on this model, we derived a new set of equations to describe our MM-EDFA:

$$
\left\{\begin{aligned}
\frac{d P_{p}(z)}{d z}=-\sigma_{a p}\left(\sum_{K} n_{1_{K}} \Gamma_{p_{K}}\right) P_{p}(z) \\
\frac{d P_{s, m}(z)}{d z}=\left(\sigma_{e s} \sum_{K} n_{2_{K}} \Gamma_{(s, m)_{K}}-\sigma_{a s} \sum_{K} n_{1_{K}} \Gamma_{(s, m)_{K}}\right) P_{s, m}(z) \\
\frac{d P_{\mathrm{ASE}_{\lambda}, m}(z)}{d z}=\left(\sigma_{e \mathrm{ASE}} \sum_{K} n_{2_{K}} \Gamma_{\left(\mathrm{ASE}_{\lambda}, m\right)_{K}}-\sigma_{a \mathrm{ASE}_{\lambda}} \sum_{K} n_{1_{K}} \Gamma_{\left(\mathrm{ASE}_{\lambda}, m\right)_{K}}\right) P_{\mathrm{ASE}_{\lambda}, m}(z) \\
+\sigma_{e \mathrm{ASE}_{\lambda}}\left(\sum_{K} n_{2_{K}} \Gamma_{\left(\mathrm{ASE}_{\lambda}, m\right)_{K}}\right) 2 h v_{\mathrm{ASE}_{\lambda}} \Delta v_{\mathrm{ASE}_{\lambda}}
\end{aligned}\right.
$$

where subscript $m$ denotes the signal mode, $K$ indicates the parameters for the $K^{\text {th }}$ ring. The variable $\Gamma$ stands for the power confinement in a given ring of a given channel in a given mode. Note that since the emission cross section is zero at $980 \mathrm{~nm}$ for the erbium ions, we omitted the term related to it. We also consider only one wavelength channel for the signal, and the background loss is neglected. The factor of 2 in Eq.(3) takes into account the two polarizations states of each mode. We also note that the azimuthal spatial dependence of the $\mathrm{LP}_{11}$ mode was dropped, $i_{m}(r, \varphi)=i_{m}(r)$, since we 
assumed that all four spatial and polarization modes average out during propagation so as to effectively yield a ring shaped intensity profile.

Along with the power evolution equations, the rate equations are given as following,

$$
\left\{\begin{array}{l}
n_{1_{K}}(z)=\frac{1 / \tau+W_{3_{K}}}{W_{1_{K}}+W_{2_{K}}+W_{3_{K}}+A_{K} / \tau} \rho_{K}(z) \\
n_{2_{K}}(z)=\frac{W_{1_{K}}+W_{2_{K}}}{W_{1_{K}}+W_{2_{K}}+W_{3_{K}}+A_{K} / \tau} \rho_{K}(z) \\
\rho_{K}(z)=n_{1_{K}}(z)+n_{2_{K}}(z)
\end{array}\right.
$$

where

$$
\left\{\begin{array}{l}
W_{1_{K}}=\frac{\sigma_{a p} \Gamma_{p_{K}} P_{p}}{h v_{p}} \\
W_{2_{K}}=\sum_{m}\left(\frac{\sigma_{a s} \Gamma_{(s, m)_{K}} P_{s, m}}{h v_{s}}+\sum_{\lambda} \frac{\left.\sigma_{a \mathrm{ASE}_{\lambda}} \Gamma_{\left(\mathrm{ASE}_{\lambda}, m\right)_{K}} P_{\mathrm{ASE}_{\lambda}, m}\right)}{h v_{\mathrm{ASE}_{\lambda}}}\right) \\
W_{3_{K}}=\sum_{m}\left(\frac{\sigma_{e s} \Gamma_{(s, m)_{K}} P_{s, m}}{h v_{s}}+\sum_{\lambda} \frac{\left.\sigma_{e \mathrm{ASE}_{\lambda}} \Gamma_{\left(\mathrm{ASE}_{\lambda}, m\right)_{K}} P_{\mathrm{ASE}_{\lambda}, m}\right)}{h v_{\mathrm{ASE}_{\lambda}}}\right)
\end{array}\right.
$$

Thus, the population density has a bi-dimensional spatial dependence along the fiber axis direction $(z)$ and the radial direction $(r)$. These equations allow us to describe with good accuracy the population dynamics and the corresponding amplification behavior of several modes, including higher-order modes.

As mentioned before, a few-mode step-index fiber of core radius $8 \mu \mathrm{m}$, cladding index 1.445 and NA $=0.1$, is assumed throughout the paper. This few-mode fiber supports two co-propagating mode groups $\left(\mathrm{LP}_{01}\right.$ and $\left.\mathrm{LP}_{11}\right)$ in the C-band.

We then consider the three types of erbium doping profiles shown in Figure 1, where the maximum relative concentration level $(\rho=1)$ corresponds to $1 \times 10^{25} \mathrm{~m}^{-3}$. We note that a minimum feature size of $0.5 \mu \mathrm{m}$ was imposed in all modeled doping profiles for fabrication tolerance.
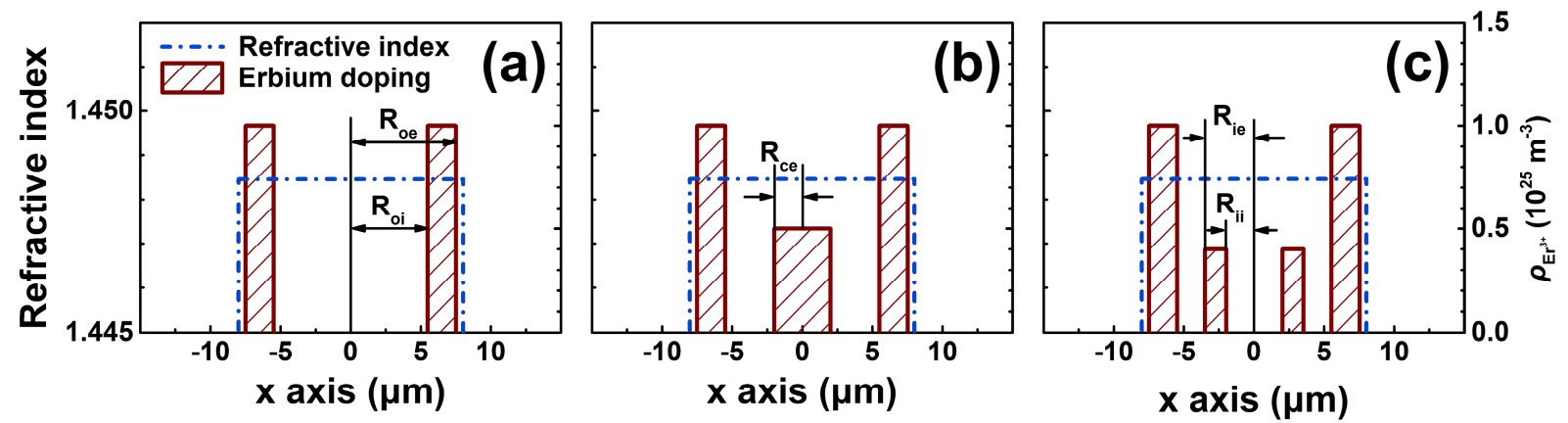

Figure 1. Erbium doping profiles: (a) outer ring, (b) outer ring + center rod, and (c) outer ring + inner ring.

A qualitative argument can be made for the doping profiles in Figure $1(b, c)$ versus that of Figure 1 (a), i.e. a single ring design. In the case of a single ring, one has limited control over the signal field overlap with the doping regions; since the $\mathrm{LP}_{01}$ mode has peak intensity in the center while the $\mathrm{LP}_{11}$ mode's peak intensities occur on both sides, a single-ring design typically favors the $\mathrm{LP}_{11}$ mode gain. By introducing multiple rings [Figure 1(c)] or a center rod [Figure 1(b)] in 
the design of the doping profile, one achieves a more precise control over the MDG. Moreover, this approach allows one to forego the complex multiple pump arrangements in favor of a single fundamental pump.

\section{SINGLE STAGE SIMULATION RESULTS}

Our doping profile design followed these steps: (i) determining and optimizing the parameters of the outer ring; (ii) adding the central doping structure, either inner ring or center rod; (iii) finding the most appropriate doping profile parameters for equalization of the MDG, and MDL compensation, in single-stage or dual-stage multimode doped amplifiers.

\subsection{Single outer ring doping: fiber parameters and analysis}

We first examined the case of a single outer ring [Figure 1(a)] of unit relative concentration, internal radius value $R_{\mathrm{oi}}$ and external radius $R_{\mathrm{oe}}$. For each pair of parameters $\left(R_{\mathrm{oi}}, R_{\mathrm{oe}}\right)$ we assessed the MM-EDFA's gain and noise characteristics by solving the two-level system rate and propagation in Equations (3)-(5) through Runge-Kutta $4^{\text {th }}$ order calculations. The simulations took into account the ASE noise power and polarization degeneracy; but neglected mode coupling effects since these were deemed small enough for the short amplifier lengths involved $(\leq 10 \mathrm{~m})$ and because the step-index profile provides weak coupling between signal modes[20]; and the fiber background loss is also neglected. Additionally, we utilized a co-propagating fundamental mode pump at $980 \mathrm{~nm}$ with $200 \mathrm{~mW}$ power.

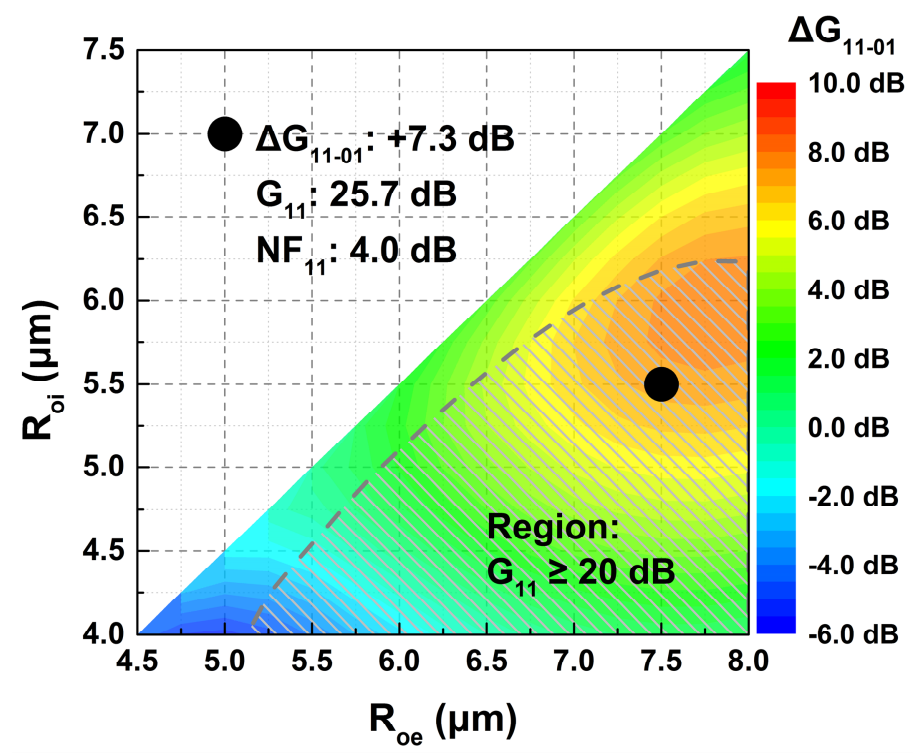

Figure 2. Differential modal gain $\left(\Delta \mathrm{G}_{11-01}\right)$ as a function of $R_{\mathrm{oi}}$ and $R_{\mathrm{oe}}$ in a $10 \mathrm{~m}$ long MM-EDFA with $200 \mathrm{~mW}$ of $980 \mathrm{~nm}$ pump. The dashed line represents the contour line of $20 \mathrm{~dB}$ gain for $\mathrm{LP}_{11}$, while more than $20 \mathrm{~dB}$ gain is obtained inside the shaded area. The black dot indicates the parameters of interest for the outer ring design.

The plot in Figure 2 of the differential gain (defined as the gain of $L P_{11}$ minus that of $L_{01}: \Delta G_{11-01}=G_{11}-G_{01}$ ) as a function of outer ring parameters $\left(R_{\mathrm{oi}}\right.$ and $\left.R_{\mathrm{oe}}\right)$ allows one to identify the region of the parameter space where $\Delta \mathrm{G}_{11-01}>0$ can be achieved with a $\mathrm{LP}_{01}$ mode forward pump at $980 \mathrm{~nm}$ wavelength and $P_{\mathrm{p}}=200 \mathrm{~mW}$ input power. We note that a MM-EDFA length of $10 \mathrm{~m}$ was implemented in the calculations of Figure 2 as we found this value to be close to the optimum length for maximum $\mathrm{LP}_{11}$ signal gain, inside the entire parameter space. Superimposed on the same figure, the shaded area indicate $\mathrm{G}_{11}>20 \mathrm{~dB}$ gain. We note that the calculated signal noise was kept below $\mathrm{NF}<5 \mathrm{~dB}$ in all our simulations. Based on these results we selected the outer ring parameters $R_{\mathrm{oi}}=5.5 \mu \mathrm{m}$ and $R_{\mathrm{oe}}=7.5 \mu \mathrm{m}$ as they yield $>$ $20 \mathrm{~dB}$ gain and a positive $\Delta \mathrm{G}_{11-01}=+7.3 \mathrm{~dB}$ gain skew for the $\mathrm{LP}_{11}$ mode, thus providing ample room for accommodating additional doping structures inside the center region that can be used to create a reciprocal negative DMG towards net gain equalization (i.e. $\Delta \mathrm{G}_{11-01}=0 \mathrm{~dB}$ ). 


\subsection{Outer ring with a central rod doping profile}

As suggested in the previous section, by adding and tuning the radius $\left(R_{\mathrm{ce}}\right)$ and concentration level $\left(\rho_{\mathrm{c}}\right)$ of a central rod in the doping profile [see Figure 1(b)] one can manage MDG and equalize the gain. Figure 3 shows the simulated DMG for the whole parameter space $\left(R_{\mathrm{ce}}, \rho_{\mathrm{c}}\right)$ while using fixed parameters $R_{\mathrm{oi}}=5.5 \mu \mathrm{m}$ and $R_{\mathrm{oe}}=7.5 \mu \mathrm{m}$ for the outer ring, which were pre-optimized in Section 3.1. The increased pump absorption due to the central doped rod resulted in the shortening of the optimum fiber length for maximum $\mathrm{LP}_{11}$ signal gain. Thus a fixed MM-EDFA length of $7 \mathrm{~m}$ was implemented in the calculations of Figure 3.

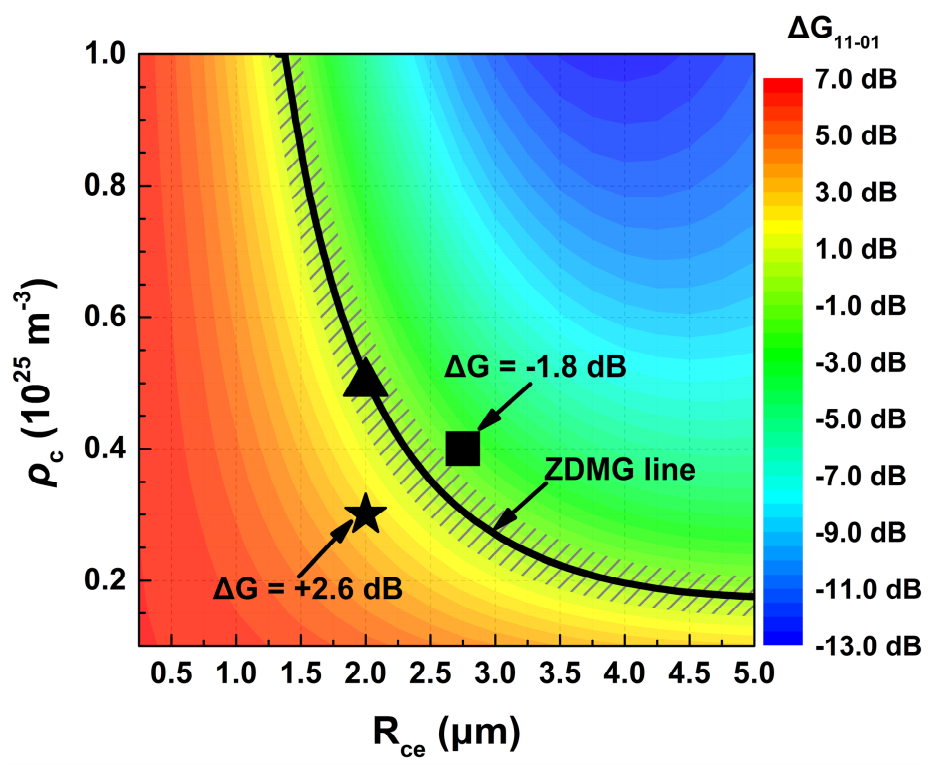

Figure 3. Differential modal gain $\left(\Delta \mathrm{G}_{11-01}\right)$ in a MM-EDFA with a fixed outer ring profile $\left(R_{\mathrm{ob}}=5.5 \mu \mathrm{m}\right.$ and $\left.R_{\mathrm{oe}}=7.5 \mu \mathrm{m}\right)$ and for varying center rod doping profiles. Pump power is $P_{\mathrm{p}}=200 \mathrm{~mW}$ and doped fiber length is $7 \mathrm{~m}$. The solid line indicates the contour line of ZDMG $(\Delta \mathrm{G}=0 \mathrm{~dB})$. The marker identified with a star corresponds to $\Delta \mathrm{G}=+2.6 \mathrm{~dB}$ while the square marker indicates $\Delta \mathrm{G}=-1.8 \mathrm{~dB}$. The shaded area surrounding the ZDMG line depicts the region where $|\Delta \mathrm{G}|<1 \mathrm{~dB}$.

The numerical results in Figure 3 indicate a variety of zero-differential modal gain (ZDMG) points, where $\Delta \mathrm{G}=0$, which forms a continuous line (solid line in Figure 3) inside the parameter space. We selected three different points denoted respectively by solid triangle, star and square markers, the first one located on ZDMG line while another two are in positive and negative regions - so as to investigate in more detail the performance of the corresponding MM-EDFA.

We remark in Figure 3 that although all points located along the ZDMG line can provide $\Delta \mathrm{G}=0 \mathrm{~dB}$, the profiles described by points located above the triangle marker are less susceptible to fluctuations in the doping concentration level of the center doped rod; while those located below the same marker are more tolerant to slight variations in the actual radius of the center doped rod. For each set of parameters identified, we first simulated the behavior of the MDG with change of input pump power as displayed in Figure 4.

Figure 4 indicates the ability of controlling the DMG by about $\pm 1 \mathrm{~dB}$ via tuning of the pump power from $150 \mathrm{~mW}$ to 300 $\mathrm{mW}$, while crossing the ZDMG line near $P_{\mathrm{p}}=200 \mathrm{~mW}$. Selecting the parameters corresponding to the solid star $\left(R_{\mathrm{ce}}=\right.$ $\left.2.0 \mu \mathrm{m}, \rho_{\mathrm{c}}=0.3\right)$ merely shifts by approximately $+2 \mathrm{~dB}$ the tuning ability with pump power. This feature enables one to dynamically adjust the modal gain in order to overcome the mode-dependent losses (MDLs) typically incurred inside a fiber span, as the $\mathrm{LP}_{01}$ mode usually suffers lower coupling losses between link components than higher-order modes. For example, if we assume input signal powers of $-13 \mathrm{dBm}$ and $-15 \mathrm{dBm}$ for the $\mathrm{LP}_{01}$ and $\mathrm{LP}_{11}$ modes at $1550 \mathrm{~nm}$ and $1555 \mathrm{~nm}$ respectively - equivalent to $\mathrm{MDL}=2 \mathrm{~dB}$ prior to amplifier - one can achieve over $18 \mathrm{~dB}$ signal gains and simultaneously compensate for the MDL by using a MM-EDFA with "solid star" doping configuration $\left(R_{\mathrm{ce}}=2.0 \mu \mathrm{m}, \rho_{\mathrm{c}}\right.$ $=0.3$ ) and input pump power: $P_{\mathrm{p}}=160 \mathrm{~mW}$ [as shown in Figure 5]. We note that slightly different wavelengths were assigned to the signal modes so as to facilitate visualization. 


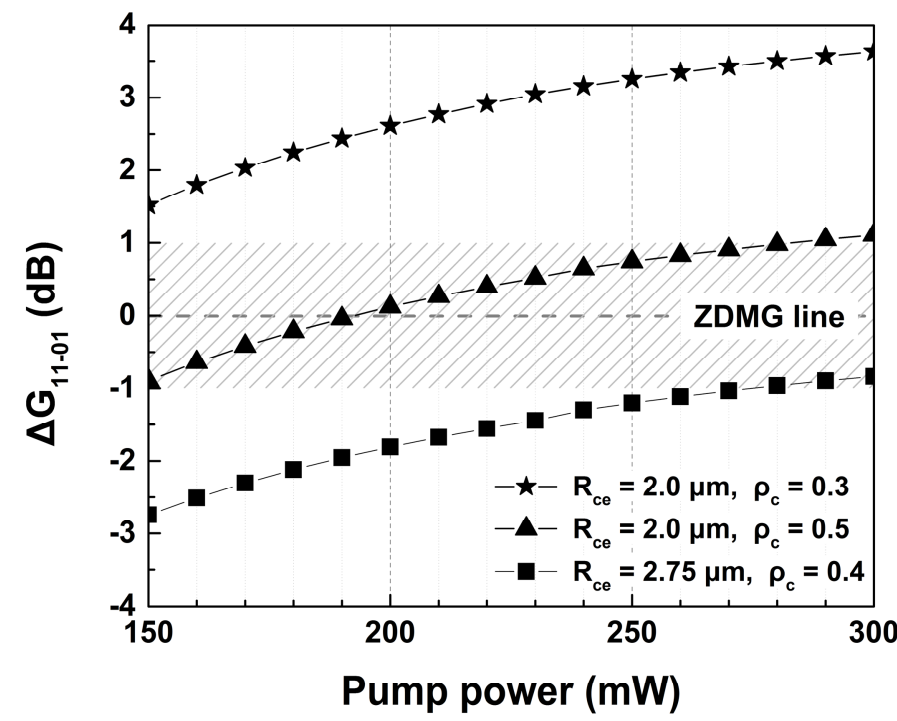

Figure 4. Differential modal gain as a function of pump power in a $7 \mathrm{~m}$ long MM-EDFA with an outer ring + center rod doping profile. The three solid markers correspond to doping profiles identified in Figure 3.

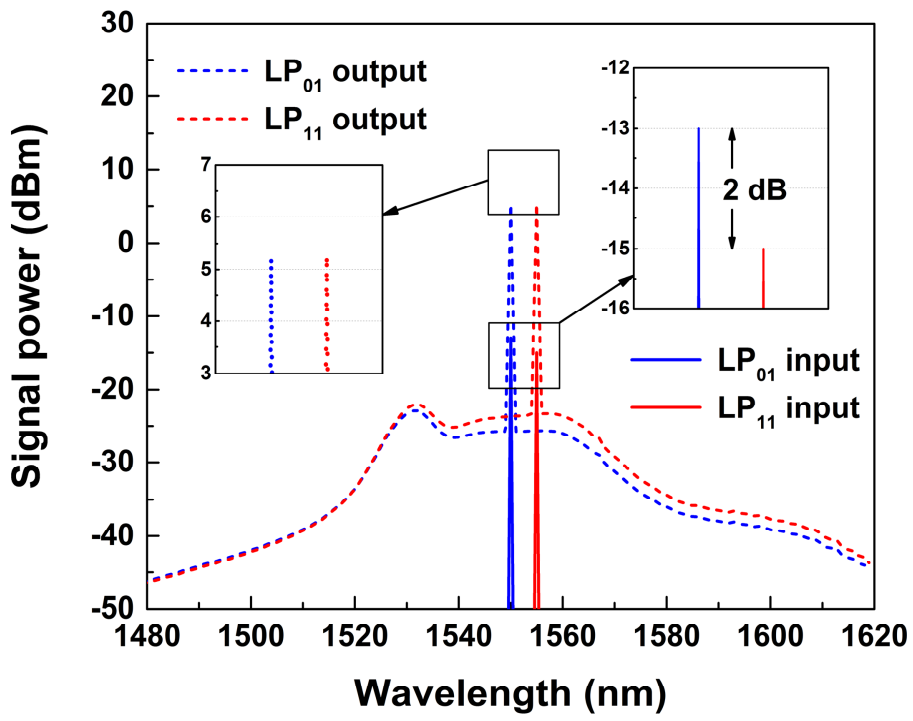

Figure 5. Input and output signal power spectra of both modes for the outer ring + center rod $\left(R_{\mathrm{ce}}=2.0 \mu \mathrm{m}, \rho_{\mathrm{c}}=0.3\right)$ doping configuration in a $7 \mathrm{~m}$ long MM-EDFA pumped with $P_{\mathrm{p}}=160 \mathrm{~mW}$ power. Insets show enlarged views of the input and output power spectra.

\subsection{Outer ring with inner ring doping profile}

Substituting the center rod for an inner doped ring [see Figure 1(c)] and keeping the parameters of the outer doped ring fixed as before $\left(R_{\mathrm{oi}}=5.5 \mu \mathrm{m}, R_{\mathrm{oe}}=7.5 \mu \mathrm{m}\right)$, parametric simulations [Figure 6] were performed as a function of the inner ring bounding radii $R_{\mathrm{ii}}$ and $R_{\mathrm{ie}}$, and using a fixed doping concentration level $\rho_{\mathrm{i}}=0.4$. The latter concentration level was found to yield adequate $\mathrm{LP}_{11}$ signal gain $\left(\mathrm{G}_{11}>20 \mathrm{~dB}\right)$ inside the whole parameter space $\left(R_{\mathrm{ii}}, R_{\mathrm{ie}}\right)$ while also providing a moderate and quasi-linear DMG response with input pump power [see Figure 7]. This systematic approach allows reducing the number of degrees of freedom to only two variables $\left(R_{\mathrm{ii}}\right.$ and $\left.R_{\mathrm{ie}}\right)$ for our parametric analysis. 


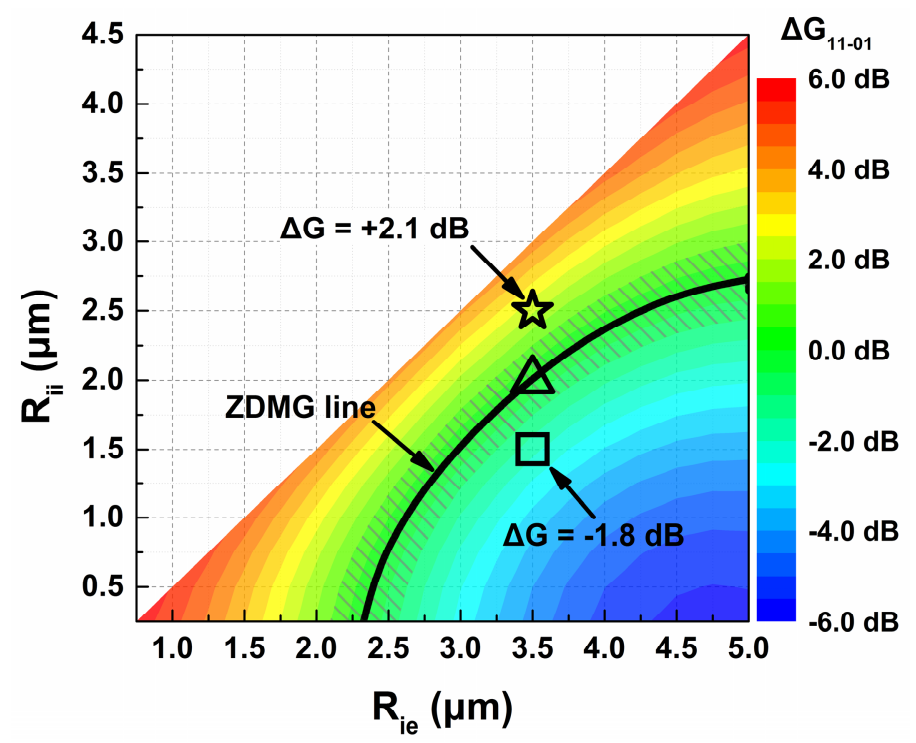

Figure 6. Differential modal gain $\left(\Delta \mathrm{G}_{11-01}\right)$ in a $7 \mathrm{~m}$ long MM-EDFA with a fixed outer ring profile $\left(R_{\mathrm{oi}}=5.5 \mu \mathrm{m}\right.$ and $R_{\mathrm{oe}}=$ $7.5 \mu \mathrm{m})$ and for varying inner ring doping profiles described with bounding radii $R_{\mathrm{ii}}$ and $R_{\mathrm{ie}}$. Pump power is $P_{\mathrm{p}}=200 \mathrm{~mW}$. The solid line indicates the contour line of ZDMG $(\Delta \mathrm{G}=0 \mathrm{~dB})$. The point identified with a hollow star corresponds to $\Delta \mathrm{G}=$ $+2.1 \mathrm{~dB}$ while hollow square indicates $\Delta \mathrm{G}=-1.8 \mathrm{~dB}$. The shaded area surrounding the ZDMG line depicts the region where $|\Delta \mathrm{G}|<1 \mathrm{~dB}$.

Based on the simulation results in Figure 6, we again selected three points of interest, denoted respectively by hollow triangle, star and square markers.

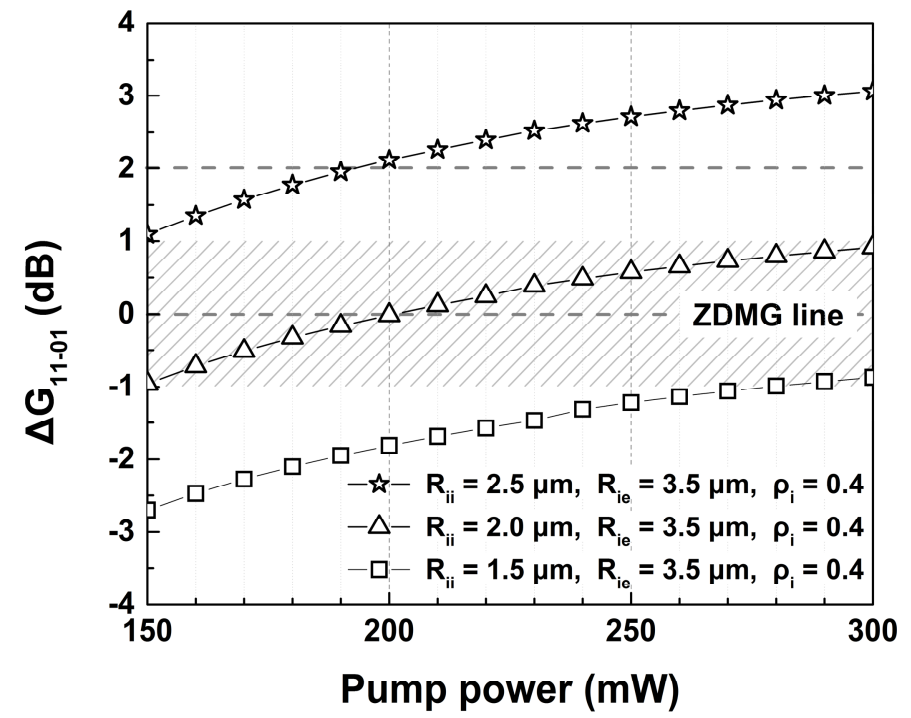

Figure 7. Differential modal gain as a function of pump power in a $7 \mathrm{~m}$ long MM-EDFA with an outer ring + inner ring doping profile.

As discussed in the previous section, the $\mathrm{LP}_{11}$ signal mode typically incurs larger losses than the $\mathrm{LP}_{01}$ mode inside a transmission link. Hence the doping profile in Figure 6 denoted by the hollow star - which provides $\Delta \mathrm{G}=+2.1 \mathrm{~dB}$ gain towards the $\mathrm{LP}_{11}$ mode - represents one example among a family of profiles that may be exploited in order to compensate for the MDL. As shown in Figure 7, the "hollow star" profile can compensate MDLs varying from 1 to $3 \mathrm{~dB}$ through a quasi-linear change of pump power from 150 to $300 \mathrm{~mW}$. Alternatively in the case of almost equal input signal powers, other doping profiles [e.g. hollow triangle marker in Figure 6] located along the ZDMG line, may similarly be 
used to obtain net gain equalization $\Delta \mathrm{G}=0 \mathrm{~dB}$ by tuning the pump power [see Figure 7]. The observed tuning sensitivity of the MDG with pump power is $\Delta \mathrm{G} / \Delta P_{\mathrm{p}} \approx 0.0013 \mathrm{~dB} / \mathrm{mW}$.

\subsection{Differential modal gain flatness study}

Besides sweeping the pump power, we also looked at the wavelength dependence for all sets of parameters highlighted earlier for both outer ring + center rod and inner ring configurations. The results for the outer ring + center rod profiles [see Figure 8] indicate that less than $1 \mathrm{~dB}$ gain excursion throughout the C-band can be achieved in case of the solid triangle marker located on the ZDMG line [see Figure 3] and for pump power levels $P_{\mathrm{p}} \geq 200 \mathrm{~mW}$. These simulations again demonstrate the possibility of achieving less than $1 \mathrm{~dB}$ modal gain excursion across the C-band by appropriate choice of multi-ring/rod doping profiles and pump power.
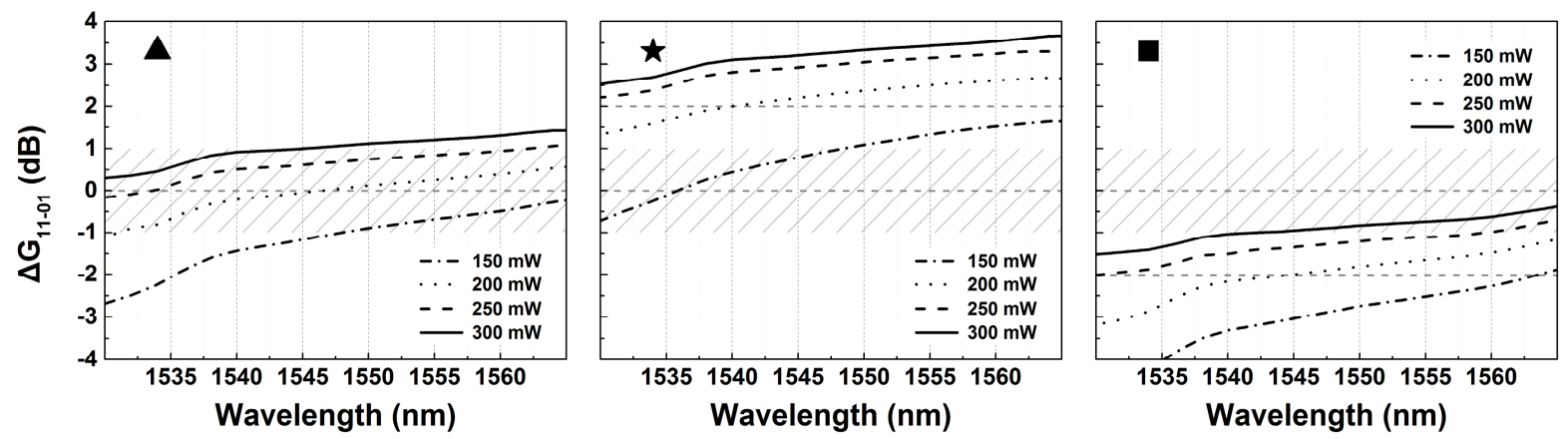

Figure 8. Differential modal gain as a function of wavelength under several pump power for the outer ring + center rod doping profile. Where the triangle, star and square marks corresponding to the same points in Figure 3 . The shaded area corresponds to the $|\Delta \mathrm{G}|<1 \mathrm{~dB}$ region.

The wavelength dependent behaviour of the DMG for the doping profiles corresponding to the three hollow markers [see Figure 6] for the outer ring + inner ring configuration [Figure 9], is analogous to that of the outer ring + center rod configuration [Figure 8]. However the MDG's wavelength dependence is noticeably flatter across the C-band for the outer ring + inner ring compared to the outer ring + center rod configuration. Especially the "hollow star" profile in Figure 9, exhibits significantly better DMG flatness with less than $0.5 \mathrm{~dB}$ gain excursion inside the C-band when $P_{\mathrm{p}} \geq$ $250 \mathrm{~mW}$.
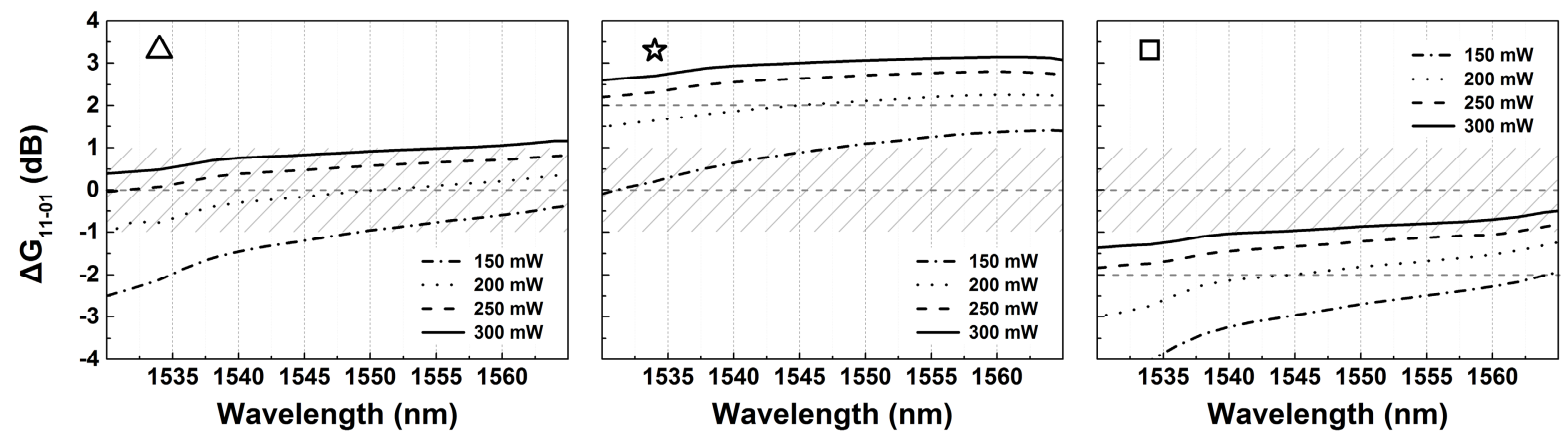

Figure 9. Differential modal gain as a function of wavelength under several pump power for the outer ring + inner ring doping profile. Where the hollow triangle, star and square marks corresponding to the same points in Figure 6. The shaded area corresponds to the $|\Delta \mathrm{G}|<1 \mathrm{~dB}$ region.

This phenomenon can be rationalized as following: as the signal wavelength goes towards long wavelengths, the intensity profile of the fundamental mode will spread and the peak intensity is subsequently reduced in the center. This is why the MDG in a MM-EDFA using a center doped rod structure is more sensitive to wavelengths shifts than a similar profile using the inner ring structure instead.

We close this section by mentioning that we also calculated the noise figures (NFs) for each signal mode in all our simulations. Typical NFs calculated for the single-stage MM-EDFAs were around $4.5 \mathrm{~dB}$ with about $0.5 \mathrm{~dB}$ noise excursions between the two signal modes. 


\section{APPLICATION IN TWO-STAGE AMPLIFIERS}

We have shown in the previous section the possibility of tuning the DMG in a single-stage configuration by varying the pump power, albeit with a small change in the signal gain. By exploiting this feature, we demonstrate in this section the ability of tuning the DMG while keeping the signal gain constant in a dual-stage configuration. Figure 10 illustrates the layout of such dual-stage MM-EDFA, where two $980 \mathrm{~nm}$ pump lasers provide fundamental mode pumping for each stage in co-direction with the input signal modes. The two pumps are combined with the signal modes by WDM couplers, and a multimode isolator is inserted to prevent backward ASE and back-reflected light from reentering the first stage. The doping profile we chose for the first stage is the outer ring + inner ring because it provides slightly lower noise figures than a comparable outer ring + center rod profile. Once the signal power levels have been substantially increased after the first stage, a second-stage with an outer ring + center rod profile enables to adjust the DMG so as to equalize and provide more signal gain.

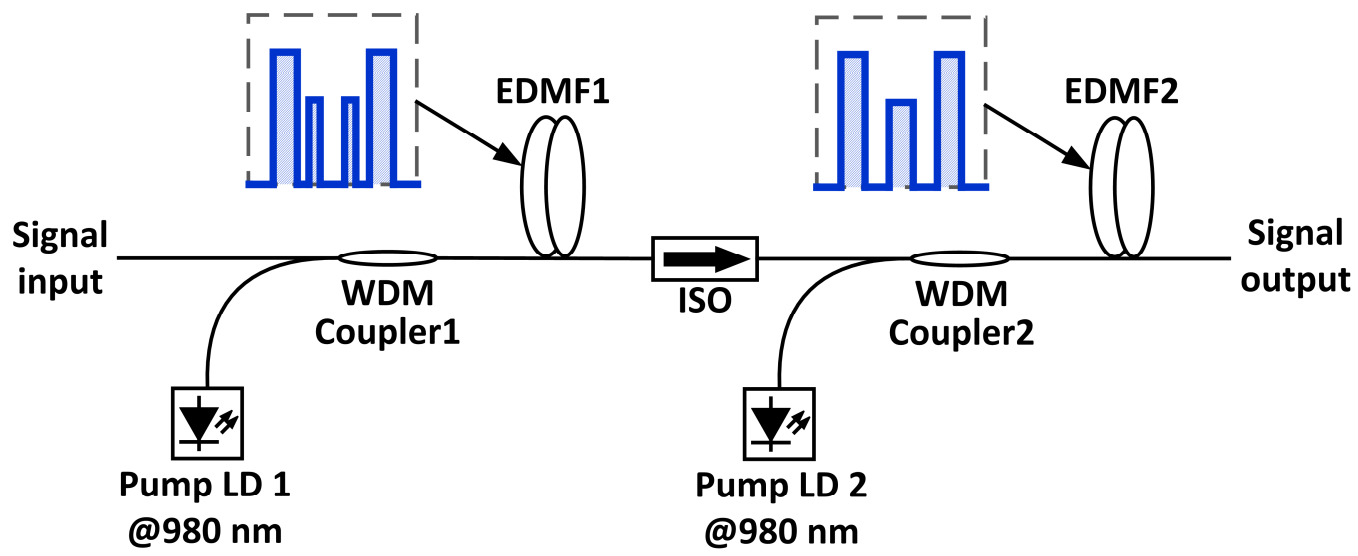

Figure 10. Scheme of two-stage MM-EDFA. Two pump lasers at $980 \mathrm{~nm}$ serve as fundamental mode forward pumping in co-propagation with the input signal. The first stage has a doping profile made of outer ring + inner ring, the second stage has an outer ring + center rod doping profile. ISO: multimode optical isolator.

We chose the hollow triangle [see Figure 6] and solid triangle [see Figure 3] doping profiles for the first stage and second stage, respectively. We set the input signal power to $-30 \mathrm{dBm}$ for both $\mathrm{LP}_{01}$ and $\mathrm{LP}_{11}$ modes, and varied the pump power for each stage. In the simulation results presented in Figure 11, we ignored coupling and isolator losses.

Figure 11 reveals that if the pump power of each stage is properly chosen along the ZDMG line (black solid line), one can equalize the DMG. Moreover, the signal gain can be fixed at either $40 \mathrm{~dB}$ or $41 \mathrm{~dB}$, for example, while the $\Delta \mathrm{G}$ is adjusted from $-0.5 \mathrm{~dB}$ to $+1.0 \mathrm{~dB}$ by proper tuning of the two-stage pump powers. The DMG tuning sensitivity with pump power is $0.013 \mathrm{~dB} / \mathrm{mW}$ for first stage and $0.007 \mathrm{~dB} / \mathrm{mW}$ for the second. Hence in this case controlling the first stage pump power effectively enables coarse tuning while that of the second stage is used for fine tuning of the DMG.

Inside a realistic transmission link, as mentioned before, the $\mathrm{LP}_{11}$ mode will typically suffer more attenuation than the fundamental mode. We here describe how to compensate such MDL by using our proposed multi-ring MM-EDFAs in a dual-stage configuration. We first select an outer ring + inner ring doping profile with positive $\mathrm{DMG}, \Delta \mathrm{G}=+2.1 \mathrm{~dB}$, (see hollow star in Figure 6) for the first stage, and an outer ring + center rod doping profile with negative DMG $\left(R_{\mathrm{ce}}=\right.$ $4.5 \mu \mathrm{m}, \rho_{\mathrm{c}}=0.25, \Delta \mathrm{G}=-2.1 \mathrm{~dB}$ ) in the second stage. The rationale for doing so is that the first stage with positive DMG acts to compensate the MDL while the second stage is used to adjust and equalize the gain. The results shown in Figure 12 , indicate a DMG tuning dynamic range from $0 \mathrm{~dB}$ to $2.5 \mathrm{~dB}$ while keeping the $\mathrm{LP}_{11}$ signal gain constant at $41 \mathrm{~dB}$. Observed tuning sensitivities for $1^{\text {st }}$ and $2^{\text {nd }}$ stages are $0.017 \mathrm{~dB} / \mathrm{mW}$ and $0.007 \mathrm{~dB} / \mathrm{mW}$, respectively.

We remark that owing to the high gain yielded by the dual-stage configuration presented previously, the noise figures of the $\mathrm{LP}_{01}$ and $\mathrm{LP}_{11}$ modes are both kept to relatively low levels of about $4 \mathrm{~dB}$ with small deviations $<0.2 \mathrm{~dB}$ between the two modes. The proposed dual-stage MM-EDFA scheme enables not only higher gain ( $>40 \mathrm{~dB})$ than a similar singlestage MM-EDFA, but also lower noise figures. 


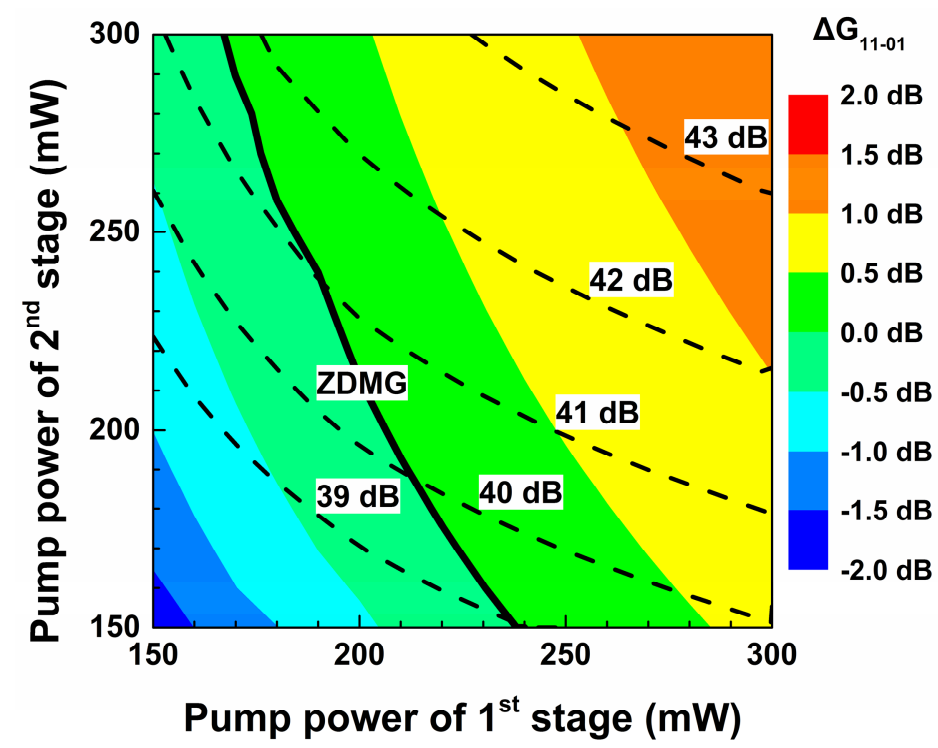

Figure 11. Differential modal gain as a function of the pump power in each stage. The black solid line indicates the pair of pump powers which can achieve ZDMG $(\Delta \mathrm{G}=0 \mathrm{~dB})$; the black dash line represent the contour line for various $\mathrm{LP}_{11}$ mode signal gain.

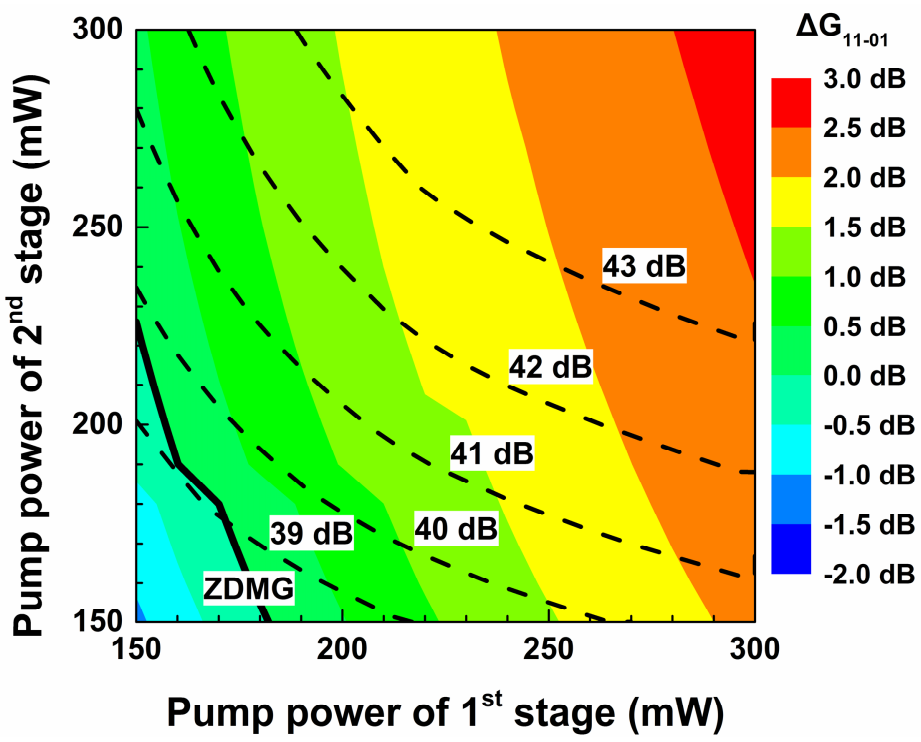

Figure 12. Differential modal gain as function matrix of the pump powers of both stages for positive and negative points configuration. The black solid line indicates the pair of pump powers which can achieve ZDMG $(\Delta \mathrm{G}=0 \mathrm{~dB})$; the black dash line represent the contour line for various $\mathrm{LP}_{11}$ mode signal gain.

\section{CONCLUSIONS}

In this paper, two types of multi-ring multi-level doping profiles for MM-EDFAs are proposed and investigated: one presents an outer ring + center rod doping profile, while the other has an outer ring + inner ring doping profile. Through numerical simulations of the single-stage MM-EDFAs performance (for $\mathrm{LP}_{01}$ and $\mathrm{LP}_{11}$ signal modes at $1550 \mathrm{~nm}$ ) using the engineered doping profiles, we demonstrated precise control over the mode-dependent gain by tuning the fundamental mode pump power (at $980 \mathrm{~nm}$ ). We predict over $20 \mathrm{~dB}$ signal gain for both modes, while gain excursion inside the C-band can be maintained below $\pm 0.8 \mathrm{~dB}$ in the case of the outer ring + center rod profile, and $\pm 0.5 \mathrm{~dB}$ for the outer ring + inner ring profile. 
In the dual-stage configuration, via independent control of the pump powers in each stage ( $150 \mathrm{~mW}$ dynamic range) we demonstrate the ability to obtain over $40 \mathrm{~dB}$ signal gain while simultaneously compensating for mode-dependent losses up to $2 \mathrm{~dB}$. Moreover, we show that the first stage may serve for coarse tuning of the DMG while the second stage may be used for fine-adjustment tuning and equalization of the DMG. Due to the high gain achieved in a dual-stage configuration, low noise figures around $4 \mathrm{~dB}$ are obtained throughout the tuning range.

Our analysis also showed that the outer ring + inner ring doping profile results in better gain flatness across the C-band, and this type of doping profile naturally favors the amplification of higher-order modes that have zero intensity in their center. Moreover, due to the circular symmetry of the multi-ring doping profiles, one is tempted to see them as potential candidates for MM-EDFAs in communication links with recently proposed fibers supporting OAM modes.

\section{REFERENCE}

[1] R. W. Tkach, "Scaling optical communications for the next decade and beyond," Bell Labs Technical Journal 14(4), 3-9 (2010) [doi:10.1002/bltj.20400].

[2] P. Krummrich and K. Petermann, "Evaluation of Potential Optical Amplifier Concepts for Coherent Mode Multiplexing," in Optical Fiber Communication Conference/National Fiber Optic Engineers Conference 2011, p. OMH5, (2011) [doi:10.1364/OFC.2011.OMH5].

[3] P. J. Winzer, "Energy-Efficient Optical Transport Capacity Scaling Through Spatial Multiplexing," IEEE Photonics Technology Letters 23(13), 851-853 (2011) [doi:10.1109/LPT.2011.2140103].

[4] S. Berdagué and P. Facq, "Mode division multiplexing in optical fibers," Applied optics 21(11), 1950-1955 (1982) [doi:10.1364/AO.21.001950].

[5] S. Murshid, B. Grossman, and P. Narakorn, "Spatial domain multiplexing: A new dimension in fiber optic multiplexing," Optics \& Laser Technology 40(8), 1030-1036 (2008) [doi:10.1016/j.optlastec.2008.03.001].

[6] R. Ryf, S. Randel, R.-J. Essiambre, and P. J. Winzer, "Space-division multiplexed transmission over few-mode-and coupled-core fiber based on coherent MIMO digital signal processing," in Proc. of SPIE ... 8284, G. Li and D. S. Jäger, Eds., pp. 828402-13 (2012) [doi:10.1117/12.915671].

[7] J. Wang, J. Yang, I. M. Fazal, et al., "Terabit free-space data transmission employing orbital angular momentum multiplexing," Nature Photonics 6(7), 488-496 (2012) [doi:10.1038/nphoton.2012.138].

[8] T. Hayashi, T. Taru, O. Shimakawa, et al., "Design and fabrication of ultra-low crosstalk and low-loss multi-core fiber," Optics Express 19(17), 16576-16592 (2011) [doi:10.1364/OE.19.016576].

[9] B. Zhu, T. F. Taunay, M. Fishteyn, et al., "112-Tb/s space-division multiplexed DWDM transmission with 14b/s/Hz aggregate spectral efficiency over a 76.8-km seven-core fiber," Optics Express 19(17), 16665-16671 (2011) [doi:10.1364/OE.19.016665].

[10] G. Nykolak, S. A. Kramer, J. R. Simpson, et al., "An erbium-doped multimode optical fiber amplifier," IEEE Photonics Technology Letters 3(12), 1079-1081 (1991) [doi:10.1109/68.118007].

[11] K.-P. Ho and J. M. Kahn, "Mode-dependent loss and gain: statistics and effect on mode-division multiplexing," Optics Express 19(17), 16612-16635 (2011) [doi:10.1364/OE.19.016612].

[12] D. Askarov and J. M. Kahn, "Design of Transmission Fibers and Doped Fiber Amplifiers for Mode-Division Multiplexing," IEEE Photonics Technology Letters 24(21), 1945-1948 (2012) [doi:10.1109/LPT.2012.2218654].

[13] E. Ip, "Gain Equalization for Few-Mode Fiber Amplifiers Beyond Two Propagating Mode Groups," IEEE Photonics Technology Letters 24(21), 1933-1936 (2012) [doi:10.1109/LPT.2012.2219521].

[14] N. Bai, E. Ip, T. Wang, and G. Li, "Multimode fiber amplifier with tunable modal gain using a reconfigurable multimode pump," Optics Express 19(17), 16601-16611 (2011) [doi:10.1364/OE.19.016601].

[15] Y. Yung, S. Alam, Z. Li, et al., "First demonstration of multimode amplifier for spatial division multiplexed transmission systems," in 37th European Conference and Exposition on Optical Communications, p. Th.13.K.4, (2011) [doi:10.1364/ECOC.2011.Th.13.K.4].

[16] R. Ryf, R. Essiambre, J. von Hoyningen-Huene, and P. Winzer, "Analysis of Mode-Dependent Gain in Raman Amplified Few-Mode Fiber," in Optical Fiber Communication Conference, p. OW1D.2, (2012) [doi:10.1364/OFC.2012.OW1D.2].

[17] G. Le Cocq, L. Bigot, A. Le Rouge, et al., "Modeling and characterization of a few-mode EDFA supporting four mode groups for mode division multiplexing," Optics Express 20(24), 27051 (2012) [doi:10.1364/OE.20.027051]. 
[18]Q. Kang, E.-L. Lim, Y. Jung, et al., "Accurate modal gain control in a multimode erbium doped fiber amplifier incorporating ring doping and a simple $\mathrm{LP}_{01}$ pump configuration.," Optics Express 20(19), 20835-20843 (2012) [doi:10.1364/OE.20.020835].

[19] E. Ip, M. Li, and C. Montero, "Experimental Characterization of a Ring-Profile Few-Mode Erbium-Doped Fiber Amplifier enabling Gain Equalization," in Optical Fiber Communication Conference/National Fiber Optic Engineers Conference 2013, p. JTh2A.18, (2013) [doi:10.1364/NFOEC.2013.JTh2A.18].

[20] Q. Kang, E. L. Lim, Y. Jung, et al., "Design of Four-Mode Erbium Doped Fiber Amplifier with Low Differential Modal Gain for Modal Division Multiplexed Transmissions," in Optical Fiber Communication Conference/National Fiber Optic Engineers Conference 2013, p. OTu3G.3, (2013) [doi:10.1364/OFC.2013.OTu3G.3].

[21] C. Giles and E. Desurvire, "Modeling erbium-doped fiber amplifiers," Journal of Lightwave Technology 9(2), 271283 (1991) [doi:10.1109/50.65886].

[22] M. Gong, Y. Yuan, C. Li, et al., "Numerical modeling of transverse mode competition in strongly pumped multimode fiber lasers and amplifiers," Optics Express 15(6), 3236 (2007) [doi:10.1364/OE.15.003236].

[23] D. Gloge, "Weakly Guiding Fibers," Applied Optics 10(10), 2252 (1971) [doi:10.1364/AO.10.002252]. 